Research Paper

\title{
Hedgehog Signaling Regulates Epithelial-Mesenchymal Transition in Pancreatic Cancer Stem-Like Cells
}

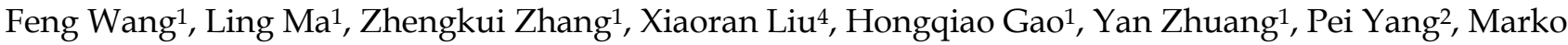 \\ Kornmann ${ }^{3}$, Xiaodong Tian ${ }^{1, 凶}$, Yinmo Yang1, ${ }^{1,}$ \\ 1. Department of General Surgery, Peking University First Hospital, Beijing 100034, People's Republic of China \\ 2. Department of Agricultural \& Biological Engineering, Purdue University, West Lafayette, IN 47906, USA \\ 3. Clinic of General, Visceral and Transplantation Surgery, University of Ulm, Ulm 89081, Germany \\ 4. Department of Breast Oncology, Peking University Cancer Hospital \& Institute, Beijing 100142, China \\ $\square$ Corresponding authors: Yinmo Yang and Xiaodong Tian, Department of General Surgery, Peking University First Hospital, 8th Xishiku Street, Beijing 100034, \\ P.R. China. Tel: +86-10-83572772. Fax: 86-10-66119730. E-mail: yangyinmosci@163.com; tianxiaodong@pkufh.cn
}

( ) Ivyspring International Publisher. Reproduction is permitted for personal, noncommercial use, provided that the article is in whole, unmodified, and properly cited. See http://ivyspring.com/terms for terms and conditions.

Received: 2015.07.22; Accepted: 2015.10.29; Published: 2016.01.20

\begin{abstract}
Hedgehog $(\mathrm{Hh})$ signaling is crucially involved in tumorigenesis. This study aimed to assess the role of $\mathrm{Hh}$ signaling in the regulation of epithelial-mesenchymal transition (EMT), stemness properties and chemoresistance of human pancreatic Panc- 1 cancer stem cells (CSCs). Panc- 1 cells were transfected with recombinant lentiviral vectors to silence SMO and serum-free floating-culture system was used to isolate Panc-1 tumorspheres. The expression of CSC and EMT markers was detected by flow cytometry, real-time RT-PCR and Western blot analysis. Malignant behaviors of Panc-1 CSC were evaluated by tumorigenicity assays and nude mouse lung metastasis model. We found that tumorspheres derived from pancreatic cancer cell line Panc-1 possessed self-renewal, differentiation and stemness properties. $\mathrm{Hh}$ pathway and EMT were active in Panc-1 tumorspheres. Inhibition of Hh signaling by SMO knockdown inhibited self-renewal, EMT, invasion, chemoresistance, pulmonary metastasis, tumorigenesis of pancreatic CSCs. In conclusion, Hh signaling contributes to the maintenance of stem-like properties and chemoresistance of pancreatic CSC and promotes the tumorigenesis and metastasis of pancreatic cancer. Hh pathway is a potential molecular target for the development of therapeutic strategies for pancreatic CSCs.
\end{abstract}

Key words: Hedgehog, pancreatic cancer, cancer stem cell, epithelial-mesenchymal transition

\section{Introduction}

Pancreatic cancer (PC) is an aggressive malignancy with a 5-year survival rate $<6 \%$ [1]. Despite recent advances in the diagnosis, surgery and chemotherapy of $\mathrm{PC}$, the prognosis of $\mathrm{PC}$ patients remains poor. At an advanced stage, $80-85 \%$ of PC patients are diagnosed when surgical resection is not feasible [2]. Therefore, understanding the molecular events underlying the development and progression of pancreatic cancer is urgently imperative to the development of novel therapeutic strategies.

Increasing evidence indicates that tumors contain a small subpopulation of cancer stem cells (CSCs) [3]. Cancer stem cells (CSCs) indicate multipotent cells exhibiting self-renewal capacity, multilineage differentiation ability and high carcinogenesis, and have been identified in several types of human cancers including pancreatic cancer [4-6]. The highly tumorigenic CSCs exhibit self-renewal by activating developmental pathways such as Wnt, Hedgehog (Hh), and Notch that participate in the maintenance of stemness [7]. As tumorigenic drivers, CSCs should be effectively targeted to achieve long-lasting therapeutic responses.

Recent studies indicate that epithelial-mesenchymal transition (EMT), a developmental process in which cells lose epithelial characteristics and acquire mesenchymal properties such as increased motility and invasion, can endow cells with stem-cell like characteristics. Hh signaling functions in the development and homeostasis of many organs 
and tissues. Aberrant activation of $\mathrm{Hh}$ signaling is associated with multifarious human tumors where the pathway is implicated in tumorigenesis, malignancy, metastasis and cancer stem cells [8]. Previous studies have shown that the activation of Hh pathway was a critical event in the tumorigenesis of pancreatic cancer $[9,10]$.

In this study we aimed to assess the role of $\mathrm{Hh}$ signaling in the regulation of EMT, stemness properties and chemoresistance of pancreatic CSCs. We isolated human pancreatic Panc- 1 tumorspheres and employed recombinant lentiviral vectors for the delivery of siRNA to knockdown SMO. We examined the expression of CSC and EMT markers and evaluated the malignant behaviors of Panc-1 CSCs in vitro and in vivo using nude mouse lung metastasis model.

\section{Materials and methods}

\section{Reagents}

Gemcitabine was obtained from Lilly. Fetal bovine serum (FBS) was from Gibeco. Antibodies for SMO, Snail, ALDH1A1 and N-cadherin were purchased from Abcam. Antibodies for E-cadherin were purchased from CST. Antibodies for Flow cytometry were purchased from Miltenyi. Cell Counting kit-8 were purchased from Dojindo, Kumamoto.

\section{Cell culture}

Pancreatic cancer cell line Panc-1 was received from the American Type Culture Collection and cultured in DMEM supplemented with $10 \%$ FBS, penicillin $(100 \mathrm{U} / \mathrm{ml})$ and streptomycin $(100 \mu \mathrm{g} / \mathrm{ml})$. A retroviral packaging cell line (ATCC 293T) was maintained in DMEM supplemented with 10\% FBS. All cell lines were maintained at $37^{\circ} \mathrm{C}$ in a humidified $5 \% \mathrm{CO} 2$ atmosphere.

\section{Lentiviral-mediated delivery of siRNA}

The RNAi targeting the human SMO gene (GenBank accession no.NM_005631.4) and the negative control sequence were designed and constructed by Invitrogen (Shanghai, China). The sequences of two different oligos targeting SMO were as follows: si-1

TGCTGTACACGATGACAAAGATGATGGTTTTGG CCACTGACTGACCATCATCTGTCATCGTGTA and CCTGTACACGATGACAGATGATGGTCAGTCAGT GGCCAAAACCATCATCTTTGTCATCGTGTAC; si-2

TGCTGTGTGCATGCTGAAGGACAGCTGTTTTGG CCACTGACTGACAGCTGTCCCAGCATGCACA and CCTGTGTGCATGCTGGGACAGCTGTCAGTC AGTGGCCAAAACAGCTGTCCTTCAGCATGCAC AC. pcDNA6.2 ${ }^{\mathrm{TM}}-\mathrm{GW} / \mathrm{EmGFP}-\mathrm{miR}$ vector was purchased from Invitrogen. miRNAs were synthesized and cloned into pcDNA6.2 $2^{\mathrm{TM}}-\mathrm{GW} / \mathrm{EmGFP}-\mathrm{miR}$, then transferred into lentiviral plasmid pLenti6.3/V5-DEST with the Gateway recombination technology. Lentiviruses were produced by the transfection of 293T cells with miRNA or negative control plasmid using packaging Mix (Invitrogen). Subconfluent cells were infected with lentivirus at a multiplicity of infection of 50 in the presence of 8 $\mathrm{ug} / \mathrm{ml}$ polybrene (Sigma Aldrich). Panc-1 cells with stable silencing of SMO were selected with $10 \mathrm{ug} / \mathrm{ml}$ of Blasticidin for 4 weeks.

Lentiviral harbouring luciferase reporter gene was generated using pBJ-luciferase, psPAX2 and pMD2.G (Addgene) in 293T packaging cells. Panc-1-NC and Panc-1-si cells were seeded in 6-well plates and transfected using the Lipofectamine system according to the manufacturer's protocol when cells achieved about $50 \%$ confluence. Cells were selected with $1 \mathrm{ug} / \mathrm{ml}$ of puromycin for 2 weeks.

\section{Real-time RT-PCR}

Total RNA was extracted from cells using TRIZOL Reagent (Invitrogen). First strand cDNA was synthesized by using the ReverTra Ace ${ }^{\circledR}$ qPCR RT kit according to the manufacturer's protocol (TOYOBO). Real-time RT-PCR was performed by using SYBR Green PCR Master Mix (TOYOBO). GAPDH was used as an internal control. The primer used were as follows: GAPDH 5'-ACGGATTTGGTCGTATTGGG-3' and 5'-TGATTTTGGAGGGATCTCGC-3'; SMO 5'-TGCTCATCGTGGGAGGCTACTT-3' and 5'-ATCT TGCTGGCAGCCTTCTCAC-3'; Gli1 5'-TATGGACCT GGCTTTGGA-3' and 5'- CCTATGTGAAGCCCTATT TGC-3'; Shh 5'-CCAATTACAACCCCGACATC-3' and 5'-CAGTTTCACTCCTGGCCACT-3'; Snail 5'-TG CCCTCAAGATGCACATCCGA-3' and 5'-GGGACA GGAGAAGGGCTTCTC-3'; E-cadherin 5'-GCCTCCT GAAAAGAGAGTGGAAG-3' and 5'-TGGCAGTGT CTCTCCAAATCCG-3'; N-cadherin 5'-TTTGATG GAGGTCTCCTAACACC-3' and 5'-ACGTTTAA CACGTTGGAAATGTG-3'; Nanog 5'-TCCTCCTCT TCCTCTATACTAAC-3' and 5'-CCCACAATCACA GGCATAG-3'. Relative quantification of gene expression was calculated on triplicate reactions using the $\triangle \triangle \mathrm{Ct}$ method. Data were analyzed by ABI 7500 software v2.0.6 (Applied Biosystems).

\section{Western blot analysis}

Cells were lysed in lysis buffer containing 1 $\mathrm{mmol} / \mathrm{L}$ Na3VO4, $1 \mathrm{mmol} / \mathrm{L}$ phenylmethylsulfonylfluoride (PMSF), $500 \mathrm{uM}$ AEBSF, $150 \mathrm{nM}$

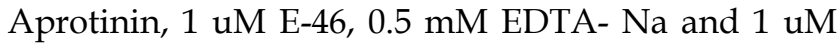
leupeptin. The lysates were cleared by centrifugation at 14,000 rpm and total protein concentration was measured with bicinchoninic acid assay Kit (Bio-Rad 
Laboratories). Cell lysates were separated in $10 \%$ SDS-PAGE and transferred onto polyvinylidene difluoride membranes (Millipore). The membranes were blocked with $5 \%$ nonfat dry milk in Tris-buffered saline with $0.1 \%$ Tween 20 , incubated with primary antibodies, and then with HRP-conjugated secondary antibodies. The peroxidase activity was detected by using Immobilon Western Chemiluminescent HRP Substrate (Millipore) and exposed to X-ray films. $\beta$-actin was used as loading control.

\section{Flow cytometry}

To detect the expression of cancer stem cell markers, the following antibodies were used: anti-CD44-PE, anti-CD24-APC, anti-ESA-PE-Vio770, IgG1-PE, IgG1-APC, IgG1-PE-Vio770 (Miltenyi). Cells were harvested with trypsin-EDTA to produce a single cell suspension. The cells were pelleted by centrifugation, washed twice with PBS and incubated with the antibody for $10 \mathrm{~min}$ in the dark at $4^{\circ} \mathrm{C}$. The respective isotype control antibodies were used at the same concentrations according to the manufacturer's instructions. After washing twice with PBS, samples were resuspended in $500 \mu \mathrm{l}$ PBS and analyzed on a flow cytometer (FACSAriaII, USA). Cells were routinely sorted twice, and the cells were reanalyzed for the purity, which typically was $>97 \%$. Data were analyzed with BD FACS Diva software.

\section{Cell proliferation assay}

The cells were plated at a density of 2,000 cells/well in 96-well plates. After exposure to the drug for $72 \mathrm{~h}$, WST-8 reagent from Cell Counting kit-8 was added ( $10 \mu \mathrm{l} /$ well), incubated for $2 \mathrm{~h}$, and optical absorbance at $450 \mathrm{~nm}$ and $630 \mathrm{~nm}$ were measured using a microplate reader. OD values of all experimental groups were normalised to control group. Inhibition rate $=(1-$ absorbance of treated cells $/$ control cells) $\times 100 \%$.

\section{Sphere formation assay}

Cells were plated in six-well ultralow attachment plates (Corning Inc.) at a density of 2,000 cells $/ \mathrm{ml}$ in serum-free DMEM:F12 (Gibco) supplemented with 20 ng/ml EGF (PeproTech), 100 ng/ml bFGF (PeproTech), 1:50 B27 supplement (Gibco), $100 \mathrm{U} / \mathrm{ml}$ penicillin, and $100 \mu \mathrm{g} / \mathrm{ml}$ streptomycin at $37^{\circ} \mathrm{C}$ in a humidified 5\% CO2 atmosphere. After 7 days of incubation, spheres were typically $>75 \mathrm{~mm}$. 7-day-old spheres were harvested using $40 \mathrm{~mm}$ cell strainers, dissociated to single cells with trypsin-EDTA, and then grown for 7 days. Differentiation was induced by culturing tumorsphere-derived cells in DMEM supplemented with $10 \%$ FBS.

\section{Transwell invasion assay}

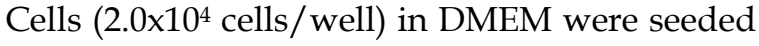
into the upper chamber of a Transwell insert (Corning), which was precoated with the Matrigel Matrix (Corning). The lower chamber was filled with DMEM supplemented with $10 \%$ of FBS as the chemoattractant. The cells were then cultured for $24 \mathrm{~h}$. The cell number was counted under a Fluorescence Inversion Microscope. Three independent experiments were performed.

\section{Tumorsphere xenografts}

Four-week-old nude mice (BALB/c-nu) were maintained in a barrier facility on high efficiency particulate air-filtered racks and fed by breeders from the Department of Laboratory Animal Science, Peking University Health Science Center. Single cells suspensions $\left(1 \times 10^{6}\right.$ in a total volume of 100 ul PBS) were injected subcutaneously into the right and left axilla of nude mice. Panc-1-NC CSCs were injected subcutaneously into left axilla and Panc-1-si CSCs was injected subcutaneously into right axilla.

Eight weeks later, all mice were sacrificed, and tumors were excised. A portion of the tumor tissue was collected, fixed in $4 \%$ paraform, and embedded in paraffin for hematoxylin and eosin (H\&E) staining to assess tumor pathology. All the animal studies were reviewed and approved by Ethics Committee for Animal Studies at the Peking University, China.

\section{Intravenous injection through vena caudalis}

Four-week-old nude mice (BALB/c-nu) were maintained in a barrier facility on high efficiency particulate air-filtered racks and fed by breeders from the Department of Laboratory Animal Science, Peking University Health Science Center. Single cells suspensions $\left(5 \times 10^{5}\right.$ in a total volume of $100 \mathrm{ul}$ of PBS) were injected into the lateral tail vein of nude mice.

\section{Statistical analysis}

Data were expressed as mean values and standard deviation (SD), and analyzed using SPSS v.13.0 statistical software (SPSS, Inc.). Student's t-test (for two groups) and one-way analysis of variance (ANOVA) test (for multiple groups) followed by the Student-Newman-Keuls (SNK) test were used to assess the differences. $\mathrm{P}<0.05$ was considered as significant difference.

\section{Results}

\section{Panc-1 tumorspheres exhibit stem-like prop- erties}

We isolated Panc-1 tumorspheres from human pancreatic cancer cell line Panc- 1 using serum-free flating-culture system. We subsequently examined 
whether Panc-1 tumorspheres have differentiation potential. ALDH1A1 and Nanog are mature markers of CSCs, and act as the differentiation markers. Real-time RT-PCR was performed to detect the expression of ALDH1A1 and Nanog. Under differentiating conditions, Panc- 1 tumorspheres began to adhere and acquired epithelium-like morphology. When returned to adherent culture, Panc- 1 tumorspheres redifferentiated and acquired morphology comparable to parental cells (Fig. 1A). We observed that Panc-1 tu-

A
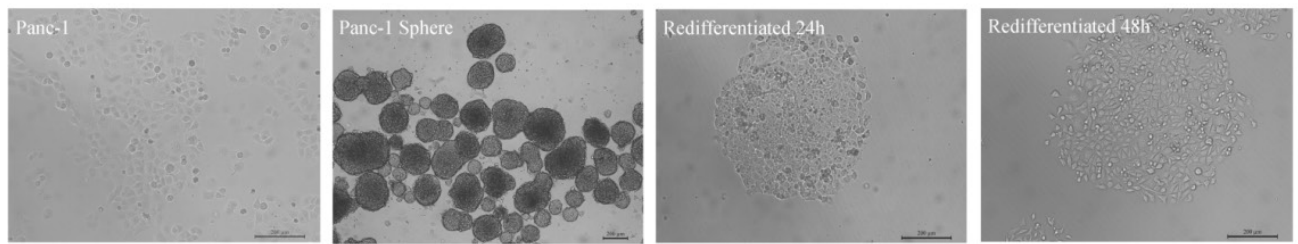

B

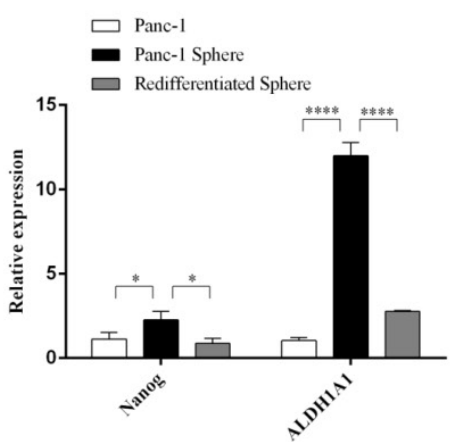

C

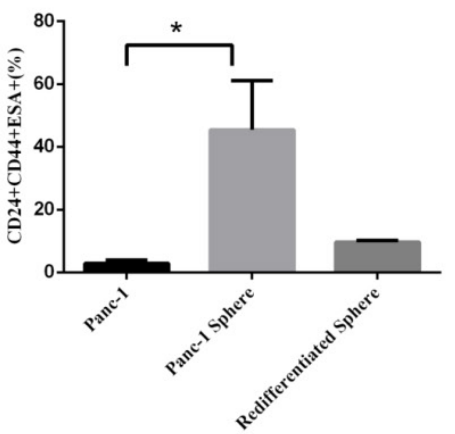

morspheres had elevated mRNA levels for genes associated with a stem-like phenotype including ALDH1A1 and Nanog that was lost upon redifferentiation (Fig. 1B). In additon, we found high percentage of Panc-1 tumorspheres CD44+CD24+ESA+ population and the percentage declined after redifferentiation (Fig.1C and D). These results suggest that Panc-1 tumorspheres have differentiation ability and stem cell-like properties.

$\mathrm{D}$
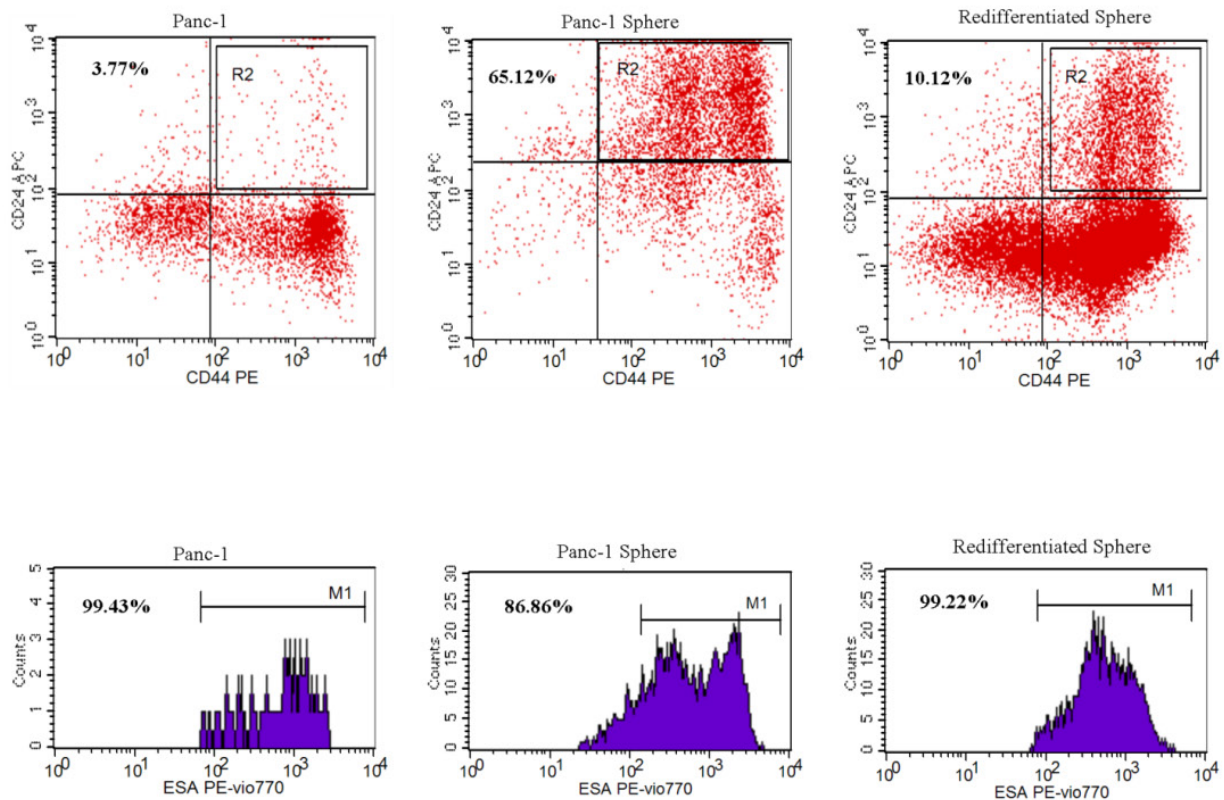

Figure 1. Differentiation ability and stem cell-like properties of Panc-1 tumorspheres. (A) The morphology of Panc-1 tumorspheres and Panc-1 re-differentiated tumorsphere. (B) The expression of Nanog and ALDHIAl was measured by Real-time RT-PCR in Panc-1, Panc-1 tumorsphere and Panc-1 re-differentiated tumorsphere. (C) The percentage of CD44+CD24+ESA+ population in Panc-1, Panc-1 tumorsphere and re-differentiated tumorsphere. (D) Flow cytometry analysis of CD24, CD44, ESA as cancer stem cell markers in Panc-1, Panc-1 tumorsphere and re-differentiated tumorsphere. Data represent mean \pm SD. $* \mathrm{P}<0.05, * * * * \mathrm{P}<0.0001$. 


\section{EMT and activated Hh signaling in Panc- 1 tu- morspheres}

Next we examined the expression of SMO, Gli1, Shh, Snail, E-Cadherin and N-Cadhern in Panc-1 tumorspheres. Real-time RT-PCR showed the high expression of SMO,Gli1 and Shh, and the activated $\mathrm{Hh}$ signaling in Panc-1 tumorspheres. In addition, we found that the expression of Snail and $\mathrm{N}$-cadherin was upregulated, while the expression of E-cadherin was down-regulation at mRNA levels in Panc-1 tumorspheres. However, after redifferentiation of Panc-1 tumorspheres SMO, Snail and N-Cadherin were downregulated while E-Cadherin was upregulated (Fig. 2).

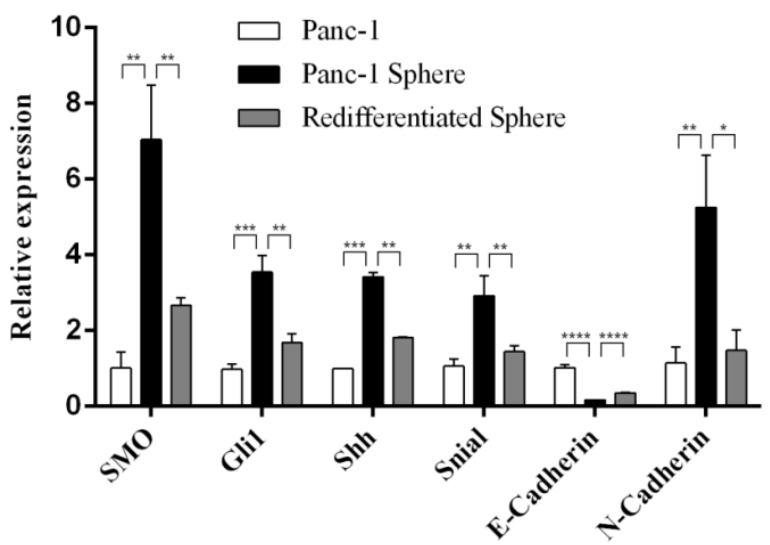

Figure 2 The expression of SMO and EMT markers in Panc-1 tumorspheres. Real-time RT-PCR analysis of the expression of SMO, Gli, Shh, Snail, E-Cadherin and N-Cadherin in Panc-1, Panc-1 tumorsphere and Panc-1 re-differentiated tumorsphere. Data represent mean $\pm \mathrm{SD}$. $* \mathrm{P}<0.05$, ** $\mathrm{P}<$ $0.01, * * * \mathrm{P}<0.001, * * * * \mathrm{P}<0.0001$.

\section{SMO regulates EMT and the expression of cancer stem cell markers in pancreatic cancer cells}

Panc-1 cells were transfected with lentiviruses for the delivery of two different siRNAs for SMO (Panc-1-si-1 and Panc-1-si-2) or negative control vectors (Panc-1-NC). Real-time RT-PCR and Western blot analysis confirmed that SMO expression was significantly downregulated in Panc-1-si cells (Fig. 3A-C). Moreover, lentivirus mediated delivery of two different siRNAs for SMO led to reduced expression of Nanog and ALDH1A1 in Panc-1 cells (Fig. 3A and C). In addition, SMO knockdown caused increased expression of E-cadherin and decreased expression of $\mathrm{N}$-cadherin and Snail (Fig. 3A-C). Finally, we detected the expression levels of cancer stem cell markers CD24CD44ESA in pancreatic cancer cells. The results showed that SMO knockdown resulted in reduced CD24+CD44+ESA+ subfraction in Panc-1-si-1 (Fig. 3D and E). Collectively, these data indicate that Hh sig- naling could regulate EMT and the expression of cancer stem cell markers in pancreatic cancer cells.

\section{SMO regulates EMT and the expression of cancer stem cell markers in pancreatic CSCs}

Panc-1-si cells were plated in six-well ultralow attachment plates in serum-free medium. Five days later, Panc-1-si tumorspheres began to form in culture. After 14 days, we found that Panc-si tumorspheres were less in number and smaller in size than Panc-1-NC tumorspheres (Fig.4A). In addition, SMO knockdown resulted in reduced percentage of CD24+CD44+ESA+ subfraction in Panc-1-si tumorsphere (Fig.4B and C).

Next we used Fluoresence-activated cell sorting (FACS) to acquire the relatively pure CD24+CD44+ESA+ Panc-1-si CSCs from Panc-1-si tumorspheres. Real-time RT-PCR and Western blot analysis confirmed that SMO expression was significantly downregulated in Panc-1-si CSCs (Fig. 4D-F). As expected, the expression of CSCs markers ALDH1A1 and Nanog was significantly downregulated in Panc-1-si CSCs (Fig. 4D-F). Furthermore, SMO knockdown in the CSCs resulted in the downregulation of epithelial marker E-cadherin and upregulation of mesenchymal markers Snail and $\mathrm{N}$-cadherin at mRNA and protein levels (Fig. 4D-F). Taken together, these results suggest that SMO is critical for the acquisition of EMT characteristics of CSCs.

\section{SMO regulates malignant behaviors of pan- creatic CSCs in vitro and in vivo}

To explore the potential role of Hh signaling in the invasive capacity of pancreatic CSCs, we employed two different siRNAs to knockdown SMO and performed Transwell invasion assay. We found that the number of Panc-1-si CSCs that migrated through the upper chamber of the Costar wells was less than that of Panc-1-NC CSCs (Fig. 5A), indicating that SMO knockdown inhibits the invasion capacity of CSCs.

Next we performed chemosensitivity assay to detect the chemotherapy resistance of Panc-1-si CSCs and Panc-1-si CSCs. Panc-1-NC CSCs were more resistant to gemcitabine than Panc-1-si CSCs (Fig. 5B). IC50 of gemcitabine was $322.33 \pm 54.72 \mu \mathrm{g} / \mathrm{ml}$ in Panc-1-NC CSCs, significantly higher than that of Panc-1-si CSCs ( $\mathrm{P}<0.05$, Fig. 5C).

Then we implanted subcutaneously equal numbers of Panc-1-NC CSC and Panc-1-si-1 CSC in nude mice and measured tumor growth. Panc-1-NC CSC had $90 \%(9 / 10)$ tumor formation while only $4 / 10$ mice injected with Panc-1-si-1 CSC showed pancreatic tumor engraftment (Fig. 5D). 
A

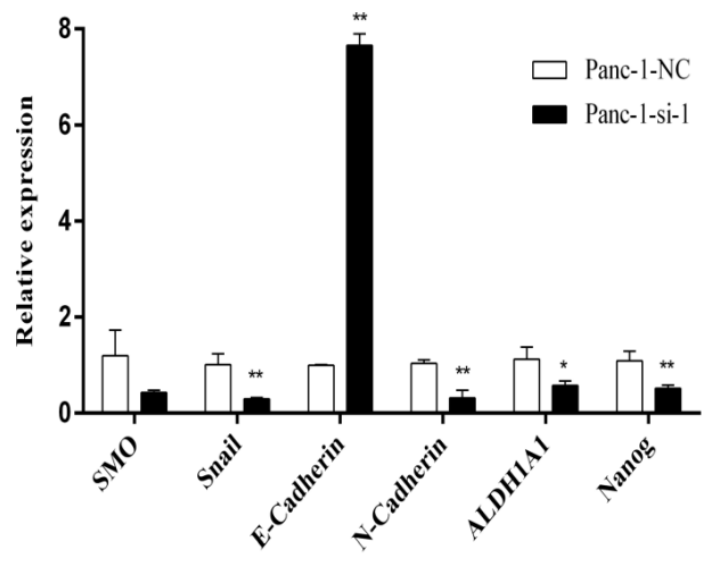

C

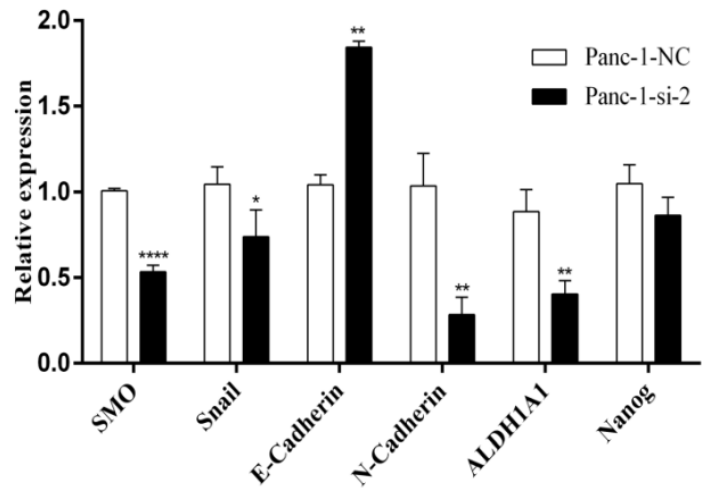

B

SMO

Snail

N-Cadherin

E-Cadherin

ALDH1A1

$\beta$-actin
Panc-1-NC Panc-1-si-1
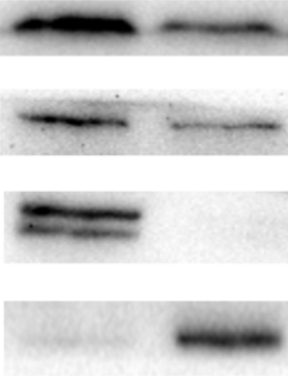
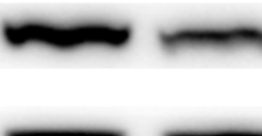

D

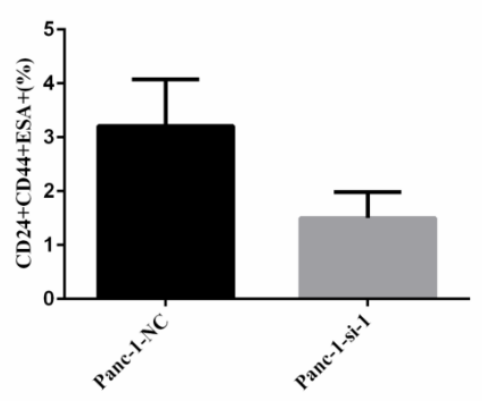

E
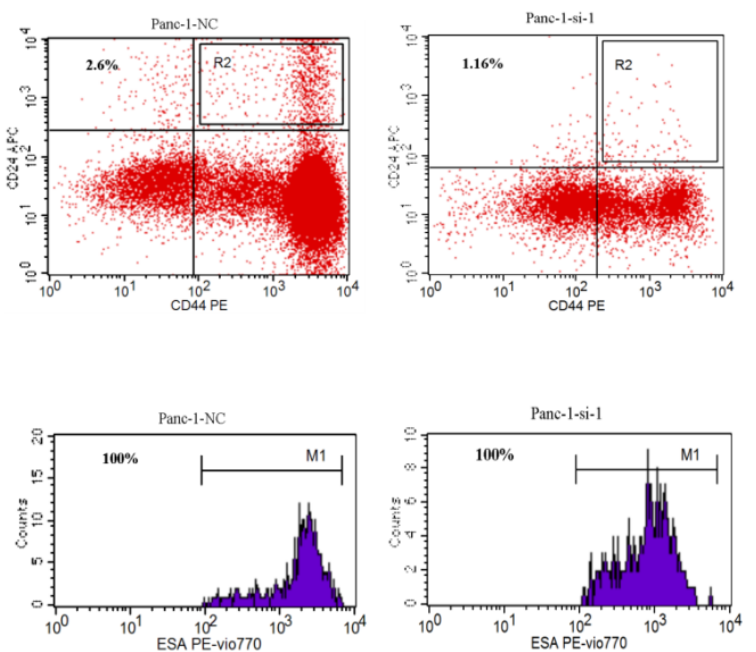

Figure 3. Changes of EMT and cancer stem cell makers after SMO silencing in Panc-1 cells. (A) Real-time RT-PCR analysis of the expression of SMO, EMT and cancer stem cell makers in Panc-1-NC and Panc-1-si-1. (B) Western blot analysis of the expression of SMO, EMT and cancer stem cell makers in Panc-1-NC and Panc-1-si-1. (C) Real-time RT-PCR analysis of the expression of SMO, EMT and cancer stem cell makers in Panc-1-NC and Panc-1-si-2. (D) The percentage of CD44+CD24+ESA+ population in Panc-1-NC and Panc-1-si-1. (E) Flow cytometry analysis of CD24, CD44, ESA as cancer stem cell markers in Panc-1-NC and Panc-1-si-1. Data represent mean \pm SD. $* \mathrm{P}<0.05$, $* * \mathrm{P}<0.01$, $* * * * \mathrm{P}<0.0001$ 
A
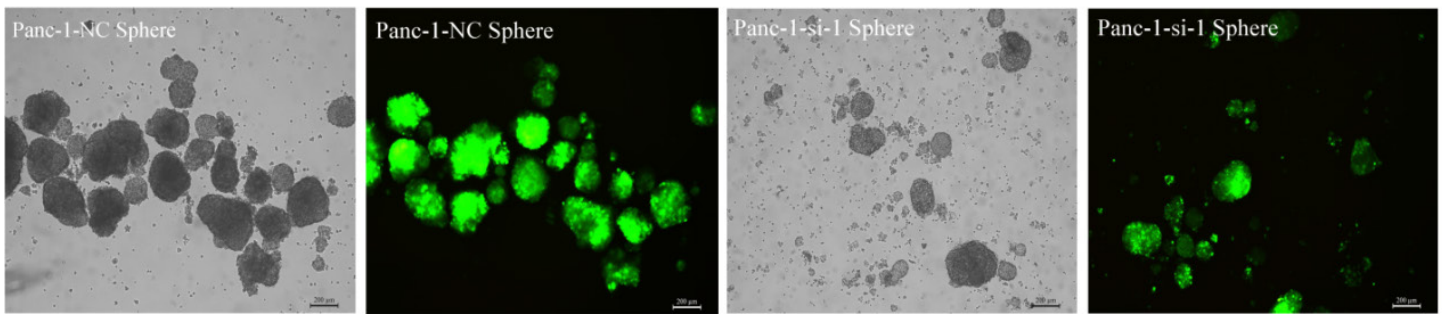

B

C
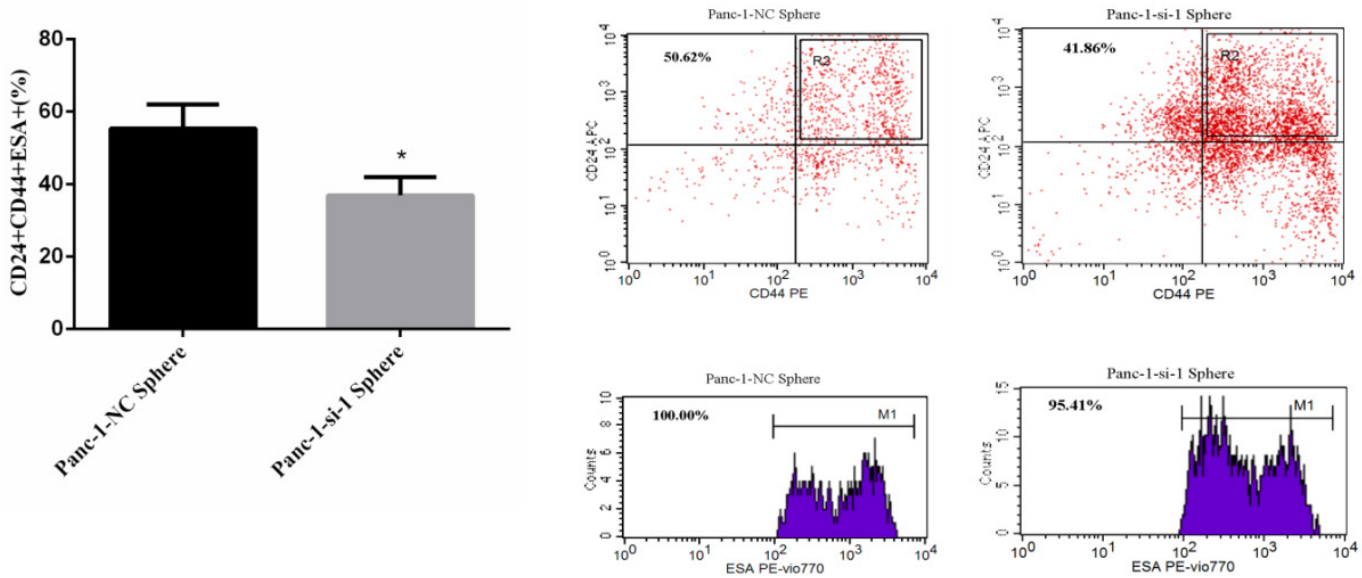

D

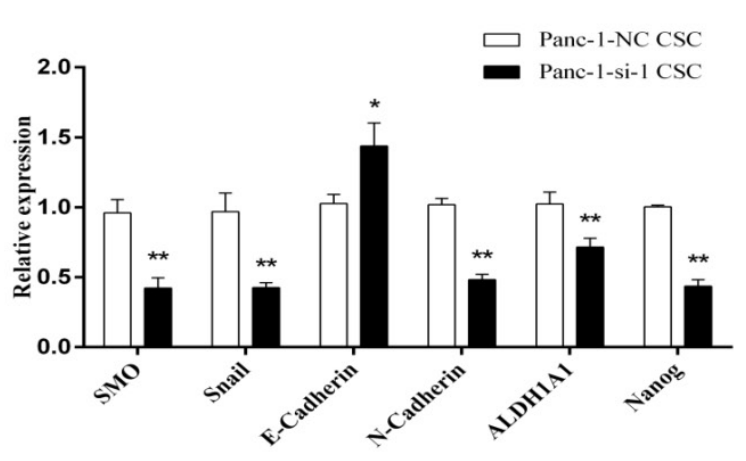

E

$\begin{array}{cc}\text { Panc-1-NC } & \text { Panc-1-si-1 } \\ \text { CSC } & \text { CSC }\end{array}$

SMO

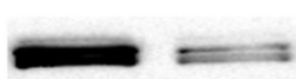

Snail

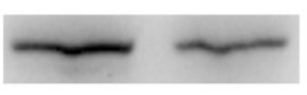

E-Cadherin

N-Cadherin

F

ALDH1Al

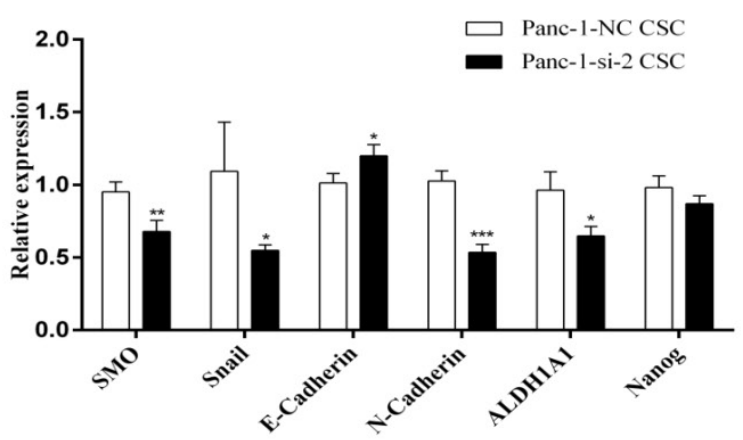

$\beta$-actin

Figure 4. Changes of EMT and cancer stem cell makers after SMO silencing in Panc-1 CSCs. (A) The morphology of Panc-1-NC and Panc-1-si-1 tumorspheres. (B) The percentage of CD44+CD24+ESA+ population in Panc-1-NC and Panc-1-si-1 tumorspheres. (C) Flow cytometry analysis of CD24, CD44, ESA in Panc-1-NC and Panc-1-si-1 tumorspheres. (D) RT-PCR analysis of SMO, EMT and cancer stem cell makers in Panc-1-NC CSCs and Panc-1-si-1 CSCs. (E) Western blot analysis of SMO, EMT and cancer stem cell makers in Panc-1-NC CSCs and Panc-1-si-1 CSCs. (F) RT-PCR analysis of SMO, EMT and cancer stem cell makers in Panc-1-NC CSCs and Panc-1-si-2 CSCs. Data represent mean \pm SD. $* P<0.05, * * P<0.01, * * * P<0.001$. 


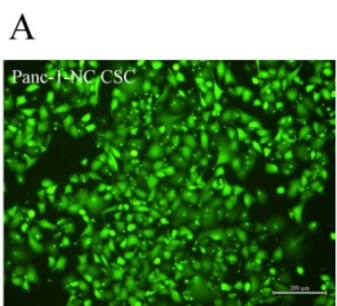

B

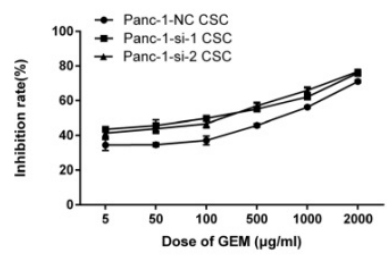

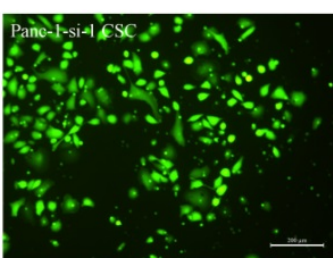

C

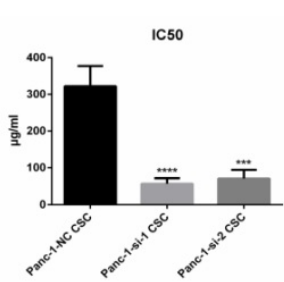

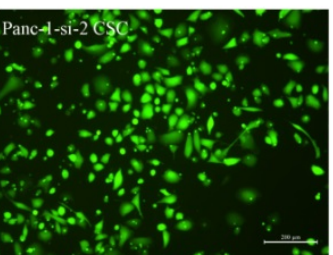

D

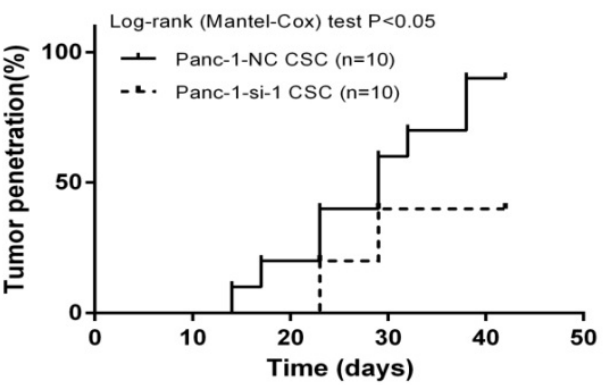

E
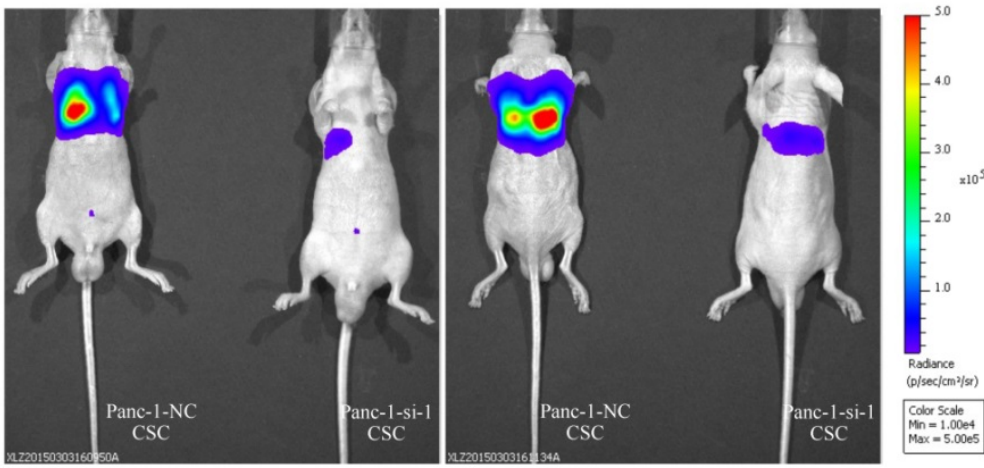

F

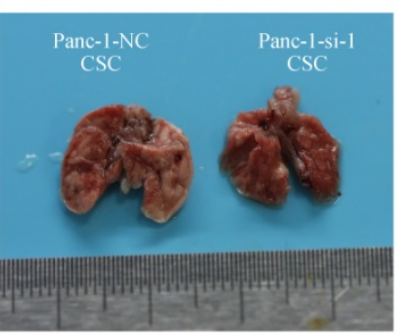

G
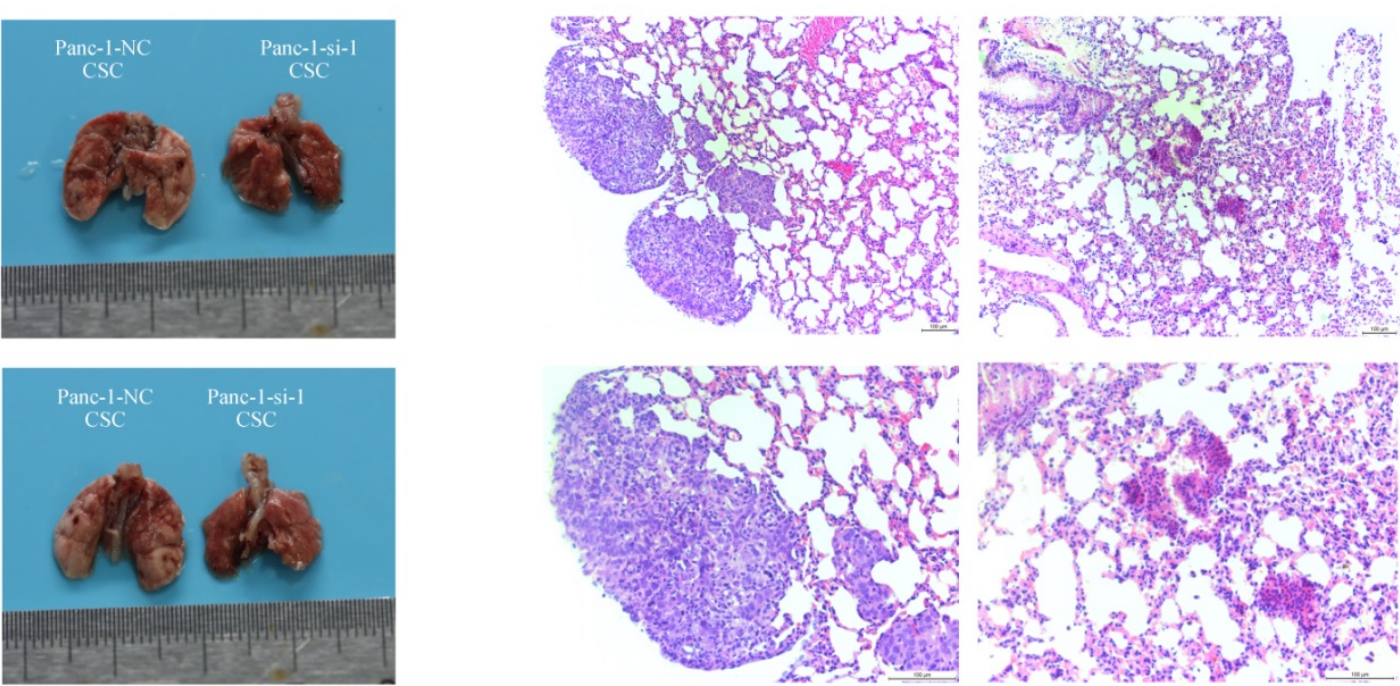

Figure 5. SMO regulates malignant behaviors of Panc-1 CSCs. (A) Effect of SMO knockdown on the invasion of Panc-1-NC CSC and Panc-1-si CSC. (B) Inhibition curve of Panc-1-NC CSC and Panc-1-si CSC at various dose of gemcitabine (GEM). (C) IC 50 of GEM in Panc-1-NC CSC and Panc-1-si CSC. (D) Tumorigenicity assay using Panc-1-NC CSC and Panc-1-si CSC. (E) The pulmonary metastasis capability of Panc-1-si CSC compared with Panc-1-NC CSC. (F) The pulmanory morphology of Panc-1-NC CSCs and Panc-1-si CSCs after intravenous injection through vena caudalis. (G) Lung metastatic lesion of Panc-1-NC CSCs and Panc-1-si CSCs. Data represent mean \pm SD. $* * * P<0.001$, ***** $\mathrm{P}<0.0001$. 
Panc-1-NC and Panc-1-si were transfected with recombinant lentiviral vectors and we used Fluoresence-activated cell sorting (FACS) to acquire the relatively pure CD24+CD44+ESA+ Panc-1-si-luci CSCs from Panc-1-si-luci tumorspheres. Single cell suspensions of Panc-1-NC-luci CSCs or Panc-1-si-luci CSCs were injected into the lateral tail vein of nude mice. We observed that Panc-1-si-luci CSCs showed reduced capability of pulmonary metastasis compared with Panc-1-NC-luci CSCs (Fig. 5E). The nude mice were killed following the lung-taken operation. Compared with control group, pulmonary metastasis was less in the test group with Panc-1-si-luci CSC intravenous injection through vena caudalis (Fig. 5F). Photomicrographs showed that tumor cells were found in 5/5 mice injected with Panc-1-NC CSCs and 0/5 mice injected with Panc-1-si-1 CSCs (Fig. 5G).

\section{Discussion}

Accumulating data indicate the existence of tumor-initiating or cancer stem cells in human pancreatic tumor that possess the capacity to self-renew and differentiate into bulk tumor cells. However, the molecular mechanisms that drive the enhanced tumorigenic potential and stem-like behavior of tumor-initiating cells are poorly understood. In this study, we provide evidence that SMO plays a vital and previously unrecognized role in the maintenance of stem-like cells isolated from human pancreatic cancer cells.

Currently, CSCs from pancreatic cancer are identified based on cell surface antigens such as CD44, CD24, epithelial-specific antigen (ESA) and CD133 [11]. There are still controversies to use which types of markers to enrich pancreatic CSCs. However, cell populations enriched for high ALDH1A1 activity were shown to be sufficient for efficient tumor-initiation with enhanced tumorigenic potential [12]. Therefore, in the present study we chose CD24CD44ESA as the surface markers of pancreatic CSCs, and the expression of ALDH1A1 and Nanog as the markers of cancer stem cell-like properties of pancreatic CSCs.

Serum-free floating culture system is an ordinary technique used to isolate CSCs from several cancer types such as brain, breast and colon cancers [13-15]. Panc-1 is a common pancreatic adenocarcinoma cell line used for the study of CSCs [16-18]. In our study, compared to control Panc-1, SMO, Snail, N-Cadherin, ALDH1A1 and Nanog were highly expressed in Panc-1 tumorsphere with decreased expression of E-cadherin and increased CD24+CD44ESA+ cell population. After the induction of differentiation, the expression levels of SMO, Snail, $\mathrm{N}$-Cadherin, E-cadherin and the CD24+CD44ESA+ cell population were similar to Panc-1. Taken together, Panc-1 tumorspheres display the potentials of self-renewal and differentiation, which are the characteristics of CSCs. Morel et al. claimed that EMT process could stimulate the stem-like capability of human breast cancer cells [19]. In this study, compared with the highly differentiated CD44-/CD24+cells, the stem cell-like CD44+/CD24cells had high expression of EMT-inducing transcription factor Snail and mesenchyme cell marker [20]. These data suggest that Hh signaling and EMT process are active in Panc- 1 tumorspheres.

Hh signaling has been associated with the self-renewal process of CSCs [21]. Previous study demonstrated that $\mathrm{Hh}$ signaling receptor SMO was highly expressed in normal human mammary stem/progenitor cells and implicated in malignant human mammary stem cells [22]. In our study, SMO knockdown resulted in the down-regulation of ALDH1A1 and Nanog and the reduction of CD24+CD44+ESA+ cell population, and reduced the size of the tumorsphere. Therefore, SMO plays a vital role in the regulation of pancreatic cancer stem cell properties and the maintenance of the self-renewal of pancreatic CSCs.

EMT is a vital process in which malignant tumor cells stem from the epithelium to obtain the capability of migration and invasion, including pancreatic tumor [23]. In this study we investigated the role of Hh signaling in EMT of pancreatic CSCs. We found that SMO knockdown resulted in declined invasion capability of Panc-1 CSCs in vitro. SMO knockdown also caused the decreases in the incidence of subcutaneous tumorigenesis and pulmonary metastasis in vivo. These data demonstrate that SMO could regulate EMT process in human pancreatic CSCs and subsequently impact the capability of invasion and migration of pancreatic CSCs.

Furthermore, EMT is implicated in the drug resistance of pancreatic tumor. Li et al. found that gemcitabine-sensitive PC cells showed strong expression of epithelial marker E-cadherin while gemcitabine-resistant PC cells exhibited strong expression of mesenchymal makers at mRNA and protein levels [24]. In addition, chemoradiation-resistant pancreatic cancer cells exhibited the properties similar to CSCs and also underwent EMT, suggesting that chemoradiation resistance-induced EMT is associated to the generation of pancreatic CSCs [25]. Thus, we wondered whether SMO could regulate the chemoresistance of pancreatic CSCs. We found that Panc-1-NC CSCs were more resistant to gemcitabine compared to Panc-1-si CSCs. These results indicate that pancreatic CSCs demonstrate declined capacity of chemoresistance with a decreased expression of SMO. 
We proposed that Hh signaling is implicated in the chemoresistance of pancreatic cancer by regulating EMT process of CSCs. Further studies in stem-like tumorspheres derived from other pancreatic cancer cell lines such as MiaPaCa-2 and L3.6pl are needed to confirm our conclusion.

In conclusion, we demonstrate that tumorspheres derived from pancreatic cancer cell line Panc-1 possess self-renewal, differentiation and stemness properties. Panc-1 tumorspheres underwent EMT and exhibited activated Hh signaling. The inhibition of Hh signaling by SMO knockdown represents an effective method to inhibit the self-renewal, EMT, invasion, chemoresistance, tumorigenesis and pulmonary metastasis of pancreatic CSCs. These data suggest that targeted therapy against Hh signaling may be beneficial in the treatment of pancreatic cancer.

\section{Acknowledgements}

This work was supported by the grants from National Natural Science Foundation of China (Grant No. 81172184 and 81372605) and Beijing Natural Science Foundation (No. 7122188, 7142162). We thank Prof. Zebin Mao at the Department of Biochemistry and Molecular Biology in Health Science Center, Peking University for the assistance and technical support.

\section{Competing Interests}

The authors have declared that no competing interest exists.

\section{References}

1. Siegel R, Desantis C, Jemal A. Colorectal cancer statistics, 2014. CA Cancer J Clin 2014;64: 104-117.

2. Vincent A, Herman I, Schulick R, Hruban RH, Goggins M. Pancreatic cancer. Lancet 2011;378: 607-620.

3. Jones RJ, Matsui WH, Smith BD. Cancer stem cells: are we missing the target. J Natl Cancer Inst 2004;96: 583-585.

4. Ye J, Wu D, Wu P, Chen Z, Huang J. The cancer stem cell niche: cross talk between cancer stem cells and their microenvironment. Tumour Biol 2014;35:3945-51.

5. Li C, Heidt DG, Dalerba P, Burant CF, Zhang L, et al. Identification of pancreatic cancer stem cells. Cancer Res 2007;67: 1030-1037.

6. Hermann PC, Huber SL, Herrler T, Aicher A, Ellwart JW, et al. Distinct populations of cancer stem cells determine tumor growth and metastatic activity in human pancreatic cancer. Cell Stem Cell 2007;1: 313-323.

7. Abetov D, Mustapova Z, Saliev T, Bulanin D. Biomarkers and signaling pathways of colorectal cancer stem cells. Tumour Biol 2015;36:1339-53.

8. Ruiz iAA. Therapeutic inhibition of Hedgehog-GLI signaling in cancer: epithelial, stromal, or stem cell targets. Cancer Cell 2008;14: 281-283.

9. Yang Y, Tian X, Xie X, Zhuang Y, Wu W, Wang W. Expression and regulation of hedgehog signaling pathway in pancreatic cancer. Langenbecks Arch Surg 2010;395: 515-525

10. Hao K, Tian XD, Qin CF, Xie XH, Yang YM. Hedgehog signaling pathway regulates human pancreatic cancer cell proliferation and metastasis. Oncol Rep 2013:29: 1124-1132.

11. Zhu J, He J, Liu Y, Simeone DM, Lubman DM. Identification of glycoprotein markers for pancreatic cancer CD24+CD44+ stem-like cells using nano-LC-MS/MS and tissue microarray. J Proteome Res 2012;11: 2272-2281.

12. Kim MP, Fleming JB, Wang H, Abbruzzese JL, Choi W, et al. ALDH activity selectively defines an enhanced tumor-initiating cell population relative to CD133 expression in human pancreatic adenocarcinoma. PLoS One 2011;6: e20636.
13. Inagaki A, Soeda A, Oka N, Kitajima H, Nakagawa J, et al. Long-term maintenance of brain tumor stem cell properties under at non-adherent and adherent culture conditions. Biochem Biophys Res Commun 2007;361: 586-592.

14. Ponti D, Costa A, Zaffaroni N, Pratesi G, Petrangolini G, et al. Isolation and in vitro propagation of tumorigenic breast cancer cells with stem/progenitor cell properties. Cancer Res 2005;65: 5506-5511.

15. Ricci-Vitiani L, Lombardi DG, Pilozzi E, Biffoni M, Todaro M, et al. Identification and expansion of human colon-cancer-initiating cells. Nature 2007;445: 111-115.

16. Gach PC, Attayek PJ, Herrera G, Yeh JJ, Allbritton NL. Isolation and in vitro culture of rare cancer stem cells from patient-derived xenografts of pancreatic ductal adenocarcinoma. Anal Chem 2013;85: 7271-7278.

17. Fredebohm J, Boettcher M, Eisen C, Gaida MM, Heller A, et al. Establishment and characterization of a highly tumourigenic and cancer stem cell enriched pancreatic cancer cell line as a well defined model system. PLoS One 2012;7: e48503.

18. Gou S, Liu T, Wang C, Yin T, Li K, et al. Establishment of clonal colony-forming assay for propagation of pancreatic cancer cells with stem cell properties. Pancreas 2007;34: 429-435.

19. Morel AP, Lievre M, Thomas C, Hinkal G, Ansieau S, Puisieux A. Generation of breast cancer stem cells through epithelial-mesenchymal transition. PLoS One 2008;3: e2888.

20. Mani SA, Guo W, Liao MJ, Eaton EN, Ayyanan A, et al. The epithelial-mesenchymal transition generates cells with properties of stem cells. Cell 2008;133: 704-715.

21. Takebe N, Harris PJ, Warren RQ, Ivy SP. Targeting cancer stem cells by inhibiting Wnt, Notch, and Hedgehog pathways. Nat Rev Clin Oncol 2011;8: 97-106.

22. Liu S, Dontu G, Mantle ID, Patel S, Ahn NS, et al. Hedgehog signaling and Bmi-1 regulate self-renewal of normal and malignant human mammary stem cells. Cancer Res 2006;66: 6063-6071.

23. Ikenaga N, Ohuchida K, Mizumoto K, Akagawa S, Fujiwara K, et al. Pancreatic cancer cells enhance the ability of collagen internalization during epithelial-mesenchymal transition. PLoS One 2012;7: e40434.

24. Li Y, VandenBoom TG 2nd, Kong D, Wang Z, Ali S, et al. Up-regulation of miR-200 and let-7 by natural agents leads to the reversal of epithelial-to-mesenchymal transition in gemcitabine-resistant pancreatic cancer cells. Cancer Res 2009;69: 6704-6712.

25. Du Z, Qin R, Wei C, Wang M, Shi C, et al. Pancreatic cancer cells resistant to chemoradiotherapy rich in "stem-cell-like" tumor cells. Dig Dis Sci 2011;56: $741-750$. 